\title{
Perturbation Theory Calculation of the Black Hole Elastic Scattering Cross Section
}

\author{
Chris Doran \\ Astrophysics Group, Cavendish Laboratory, Madingley Road, \\ Cambridge CB3 0HE, UK.
}

\begin{abstract}
The differential cross section for scattering of a Dirac particle in a black hole background is found. The result is the gravitational analog of the Mott formula for scattering in a Coulomb background. The equivalence principle is neatly embodied in the cross section, which depends only on the incident velocity, and not the particle mass. The low angle limit agrees with classical calculations based on the geodesic equation. The calculation employs a well-defined iterative scheme which can be extended to higher orders. Repeating the calculation in different gauges shows that our result for the cross section is gauge-invariant and highlights the issues involved in setting up a sensible iterative scheme.
\end{abstract}

PACS numbers: 11.80.-m, 03.80.+r, 04.70.Bw, 04.62.+v

\section{Introduction}

Scattering of a charged fermion in a background Coulomb field is a widely studied process which can be analysed perturbatively in quantum field theory. To lowest order the scattering process is summarised in the Mott formula for the unpolarised differential scattering cross section [1],

$$
\left.\frac{d \sigma}{d \Omega}\right|_{\text {Mott }}=\frac{Z^{2} \alpha^{2}}{4 \boldsymbol{p}^{2} v^{2} \sin ^{4}(\theta / 2)}\left(1-v^{2} \sin ^{2}(\theta / 2)\right) .
$$

Here $\boldsymbol{p}$ is the momentum, $v=|\boldsymbol{p}| / E, \alpha$ is the fine structure constant and the source has charge $Z e$. Curiously, there have only been sporadic attempts to repeat the analysis leading to the Mott formula for the case of a black hole [2, 3]. The problem of scattering by a black hole has certainly been tackled by many authors (see for example the books by Futterman, Handler and Matzner [4] and Chandrasekhar [5], or the recent article by Andersson and Jensen [6]). But few authors tackle the problem in perturbation theory and there is a notable reluctance to analyse the fermion case, with most work carried out for the case of a massless scalar field.

Unlike scattering in a Coulomb field, black hole scattering is complicated by the additional effects of absorption and emission. Absorption is due to the singularity in the gravitational field and manifests itself as a lack of Hermiticity in the fermion wave equation [7, 8, 9]. Emission is due to the Hawking radiation and cannot effectively be treated without some of the apparatus of quantum field theory [10]. Despite these complicating factors, we show here that an iterative

\footnotetext{
${ }^{1}$ e-mail: c.doran@mrao.cam.ac.uk, http://www.mrao.cam.ac.uk/ cjld1/

${ }^{2}$ e-mail: a.n.lasenby@mrao.cam.ac.uk
} 
scheme can be set up, based on the Dirac equation, which produces a formula for the lowest-order scattering cross section with little difficulty.

In this paper we concentrate on the scattering cross section for a fermion in the background field of a spherically-symmetric black hole. We find that the unpolarised differential scattering cross section is given by

$$
\frac{d \sigma}{d \Omega}=\frac{(G M)^{2}}{4 v^{4} \sin ^{4}(\theta / 2)}\left(1+2 v^{2}-3 v^{2} \sin ^{2}(\theta / 2)+v^{4}-v^{4} \sin ^{2}(\theta / 2)\right) .
$$

We calculate this result in two quite different gauges, which require going to different orders in perturbation theory. The first calculation utilises a series of gauge choices for the gravitational fields which convert the Dirac equation into a simple, Hamiltonian form [8, 9]. This can be analysed straightforwardly through a perturbation expansion. Unlike approaches based on the Schwarzschild coordinate system, the gauge used here does not contain a singularity at the horizon and extends right up to the origin. This means that each step in the perturbation series can be treated exactly, avoiding the problems encountered by Collins et al. [2] who attempted a Born approximation scheme based on Schwarzschild coordinates. The result they obtained was physically unreasonable, though their explanation of why this occurred (as the result of wavepacket dispersion) seems incorrect in light of the present calculation.

The choice of gauge employed in our first calculation leads to an unusual form of the vertex factor in momentum space, which vanishes when both the incoming and outgoing fermions are on-shell. It follows that the first-order contribution to the scattering cross section in this gauge is identically zero. Since the vertex factor turns out to go as the square root of the black hole mass, the fact that the process is second-order does make sense. The integrals involved in the second-order calculation are all finite and do not require any form of regularisation procedure. The result of the calculation (2) is the gravitational analog of the Mott scattering formula. For low energies the cross section reduces to the familiar Rutherford formula. As the energy is increased, relativistic corrections become more significant. The formula neatly embodies the equivalence principle, in that the cross-section depends only on the particle's velocity, and not its mass. In the low-angle limit our formula agrees with earlier results for the classical cross-section based on the geodesic equation [2].

The success of the calculation suggests a more general scheme for tackling the scattering problem, and this is explored in the second half of this paper. We calculate the cross section in a different gauge, using a first-order scheme, and confirm that the same result is obtained. This verifies that the cross-section formula is gauge-invariant. The second calculation also highlights the conceptual difficultly of carrying through a full programme based on perturbation theory, as choice of gauge at the start of the calculation can dictate features as important as the order of Feynman diagrams one needs to consider. This appears to be the correct explanation for the failure of the Collins et al. [2] calculation. Their scheme was based on the Schwarzschild gauge, and they only went to first order in perturbation theory. But to be consistent we should include terms from the Schwarzschild metric to first order in $M$, which then recovers the correct result.

Throughout we employ units with $c=\hbar=1$, while factors of $G$ are stated explicitly. Where appropriate, factors of $c$ and $\hbar$ are also included. The Minkowski spacetime metric has signature $(1,-1,-1,-1)$. 


\section{The Dirac Equation}

Our starting point is the Schwarzschild line element in its standard form

$$
d s^{2}=\left(1-\frac{2 G M}{r}\right) d \vec{t}^{2}-\left(1-\frac{2 G M}{r}\right)^{-1} d r^{2}-r^{2}\left(d \theta^{2}+\sin ^{2} \theta d \phi^{2}\right),
$$

where $\bar{t}$ is the proper time measured by stationary observers, and $r, \theta$ and $\phi$ have their usual meaning. We first transform to a new time coordinate $t$, corresponding to the proper time for particles infalling radially from rest at infinity. This coordinate has

$$
d t=d \bar{t}+\frac{(2 G M r)^{1 / 2}}{r-2 G M} d r .
$$

In terms of this new coordinate the line element (3) becomes

$$
d s^{2}=d t^{2}-\left(d r+\left(\frac{2 G M}{r}\right)^{1 / 2} d t\right)^{2}-r^{2}\left(d \theta^{2}+\sin ^{2} \theta d \phi^{2}\right) .
$$

The coordinate system is valid for $0 \leq r<\infty$. We will not consider the effects of analytic continuation through the introduction of Kruskal-Szekeres coordinates. See [8, 9] for a more detailed discussion of the form of the metric employed here.

In order to write the Dirac equation in its simplest form it is useful to revert to Cartesian coordinates by writing

$$
\begin{aligned}
& x=r \sin \theta \cos \phi \\
& y=r \sin \theta \sin \phi \\
& z=r \cos \theta .
\end{aligned}
$$

With the coordinates written $x^{\mu}=(t, x, y, z), \mu=0 \ldots 3$, the line element (5) can be written as

$$
d s^{2}=\eta_{\mu \nu} d x^{\mu} d x^{\nu}-\frac{2 G M}{r} d t^{2}-\frac{2}{r}\left(\frac{2 G M}{r}\right)^{1 / 2} x^{i} d x^{i} d t
$$

where $i=1 \ldots 3, \eta_{\mu \nu}$ is the Minkowski metric and repeated indices are summed over. To write down the Dirac equation in this background we start with the standard Dirac $\gamma$-matrices and from these we define the sets $\left\{g^{\mu}(x)\right\}$ and $\left\{g_{\mu}(x)\right\}$ by

$$
g^{0}=\gamma^{0}, \quad g^{i}=\gamma^{i}-\left(\frac{2 G M}{r}\right)^{1 / 2} \frac{x^{i}}{r} \gamma^{0}
$$

and

$$
g_{0}=\gamma^{0}+\left(\frac{2 G M}{r}\right)^{1 / 2} \frac{x^{i}}{r} \gamma_{i}, \quad g_{i}=\gamma_{i}
$$

It is straightforward to check that these satisfy

$$
\left\{g_{\mu}, g_{\nu}\right\}=2 g_{\mu \nu} I, \quad\left\{g_{\mu}, g^{\nu}\right\}=2 \delta_{\mu}^{\nu} I
$$


where $g_{\mu \nu}$ is the metric specified by (7) and $I$ is the identity matrix. In terms of the $g^{\mu}$ matrices the Dirac equation is

$$
i g^{\mu} \nabla_{\mu} \psi=m \psi
$$

where

$$
\nabla_{\mu} \psi=\left(\partial_{\mu}+\frac{i}{2} \Gamma_{\mu}^{\alpha \beta} \Sigma_{\alpha \beta}\right) \psi, \quad \Sigma_{\alpha \beta}=\frac{i}{4}\left[\gamma_{\alpha}, \gamma_{\beta}\right],
$$

and the components of the spin connection are found in the standard way (see [11], for example). With our choice of matrices we find that the Dirac equation takes the simple form

$$
i \not \partial \psi-i \gamma^{0}\left(\frac{2 G M}{r}\right)^{1 / 2}\left(\frac{\partial}{\partial r}+\frac{3}{4 r}\right) \psi=m \psi .
$$

Here $\not \partial=\gamma^{\mu} \partial_{\mu}$ is the familiar Dirac derivative operator in Minkowski spacetime. The gravitational effects are contained in a single interaction term in an analogous manner to the Coulomb interaction. The main difference is the presence of a radial derivative. This form of the equation is clearly ideal for scattering calculations as the interaction can be treated perturbatively. In the asymptotic region the metric coordinates all agree with the standard Minkowski interpretation, so there is no ambiguity in the meaning of any cross sections computed.

\section{Non-Relativistic Approximation}

Before studying the relativistic cross section it is useful to first consider the nonrelativistic limit of the Dirac equation. The Hamiltonian form of (13) contains a single interaction term

$$
\hat{H}_{I} \psi=i \hbar(2 G M / r)^{1 / 2} r^{-3 / 4} \partial_{r}\left(r^{3 / 4} \psi\right),
$$

where dimensional constants have been included. This interaction Hamiltonian, which incorporates all (general) relativistic effects, is independent of the particle mass and so embodies the equivalence principle. The interaction term is also independent of the speed of light, so the non-relativistic approximation of the

Dirac equation can proceed in the standard manner [1]. There are no spin effects to consider, so we arrive at the Schrödinger equation

$$
-\frac{\hbar^{2}}{2 m} \nabla^{2} \psi+i \hbar(2 G M / r)^{1 / 2} r^{-3 / 4} \partial_{r}\left(r^{3 / 4} \psi\right)=E \psi
$$

where we have assumed that we have a stationary state of energy $E$. To simplify this equation we introduce the phase-transformed variable

$$
\Psi=\psi \exp \left(-i\left(r / a_{G}\right)^{1 / 2}\right)
$$

where

$$
a_{G}=\frac{\hbar^{2}}{8 G M m^{2}}
$$


and is (eight times) the gravitational equivalent of the Bohr radius. The new variable $\Psi$ satisfies the simple equation

$$
-\frac{\hbar^{2}}{2 m} \nabla^{2} \Psi-\frac{G M m}{r} \Psi=E \Psi .
$$

This is precisely the equation we would expect if we used the Newtonian gravitational potential, and the solutions for $\Psi$ are Coulomb wave functions. The standard arguments about the long range logarithmic phase effects in Coulomb wave functions apply equally to the $r^{1 / 2}$ behaviour, so the cross section can be found in the conventional way [12]. So, in the non-relativistic limit, the gravitational differential scattering cross section reduces to the Rutherford formula. We therefore expect that the full, relativistic calculation will give a cross section which reduces to the Rutherford formula for small velocities.

\section{Scattering Cross Section}

The Dirac equation (13) is well-suited to an iterative solution in the standard manner. We seek a solution of

$$
\left[i \not \partial_{2}-B\left(x_{2}\right)-m\right] S_{G}\left(x_{2}, x_{1}\right)=\delta^{4}\left(x_{2}-x_{1}\right)
$$

where

$$
B(x)=i \gamma^{0}\left(\frac{2 G M}{r}\right)^{1 / 2}\left(\frac{\partial}{\partial r}+\frac{3}{4 r}\right)
$$

The iterative solution to this equation is

$$
\begin{aligned}
& S_{G}\left(x_{f}, x_{i}\right)=S_{F}\left(x_{f}, x_{i}\right)+\int d^{4} x_{1} S_{F}\left(x_{f}, x_{1}\right) B\left(x_{1}\right) S_{F}\left(x_{1}, x_{i}\right) \\
& +\iint d^{4} x_{1} d^{4} x_{2} S_{F}\left(x_{f}, x_{1}\right) B\left(x_{1}\right) S_{F}\left(x_{1}, x_{2}\right) B\left(x_{2}\right) S_{F}\left(x_{2}, x_{i}\right)+\cdots
\end{aligned}
$$

where $S_{F}\left(x_{2}, x_{1}\right)$ is the (position space) Feynman propagator. The interaction term $B(x)$ is independent of time so energy is conserved throughout the interaction. Converting to momentum space we define the amplitude

$$
\mathcal{M}=\bar{u}_{s}\left(\boldsymbol{p}_{f}\right) V u_{r}\left(\boldsymbol{p}_{i}\right)
$$

where

$$
V=B\left(\boldsymbol{p}_{f}, \boldsymbol{p}_{i}\right)+\int \frac{d^{3} k}{(2 \pi)^{3}} B\left(\boldsymbol{p}_{f}, \boldsymbol{k}\right) \frac{\not k+m}{k^{2}-m^{2}+i \epsilon} B\left(\boldsymbol{k}, \boldsymbol{p}_{i}\right)+\cdots
$$

Here $B\left(\boldsymbol{p}_{f}, \boldsymbol{p}_{i}\right)$ is the spatial Fourier transform of the interaction term, bold symbols refer to spatial components only, and for the spinor terms we follow the conventions of Mandl and Shaw 13. In terms of $\mathcal{M}$ the differential cross section is given by

$$
\frac{d \sigma}{d \Omega}=\left(\frac{m}{2 \pi}\right)^{2}|\mathcal{M}|^{2}
$$


The Fourier transform of the interaction term is

$$
B\left(\boldsymbol{p}_{2}, \boldsymbol{p}_{1}\right)=(2 G M)^{1 / 2} i \gamma^{0} \int d^{3} x e^{-i \boldsymbol{p}_{2} \cdot \boldsymbol{x}} \frac{1}{r^{1 / 2}}\left(\frac{\partial}{\partial r}+\frac{3}{4 r}\right) e^{i \boldsymbol{p}_{1} \cdot \boldsymbol{x}}
$$

where bold symbols refer to spatial components only. To evaluate this we first write

$$
B\left(\boldsymbol{p}_{2}, \boldsymbol{p}_{1}\right)=(2 G M)^{1 / 2} i \gamma^{0}\left(\frac{3}{4} f\left(\boldsymbol{p}_{1}-\boldsymbol{p}_{2}\right)+\left.\frac{\partial f\left(\lambda \boldsymbol{p}_{1}-\boldsymbol{p}_{2}\right)}{\partial \lambda}\right|_{\lambda=1}\right)
$$

where

$$
f(\boldsymbol{p})=\int d^{3} x \frac{e^{i \boldsymbol{p} \cdot \boldsymbol{x}}}{r^{3 / 2}}=\left(\frac{2 \pi}{|\boldsymbol{p}|}\right)^{3 / 2} .
$$

We therefore find that the momentum space vertex factor is

$$
B\left(\boldsymbol{p}_{2}, \boldsymbol{p}_{1}\right)=3 \pi^{3 / 2} i(G M)^{1 / 2} \frac{\boldsymbol{p}_{2}{ }^{2}-\boldsymbol{p}_{1}{ }^{2}}{\left|\boldsymbol{p}_{2}-\boldsymbol{p}_{1}\right|^{7 / 2}} \gamma^{0} .
$$

This vertex factor has the unusual feature of vanishing if the ingoing and outgoing particles are on-shell, since energy is conserved. It follows that the lowest order contribution to the scattering cross section vanishes. This is reassuring, as the vertex factor goes as $\sqrt{M}$, and we expect the amplitude to go as $M$ to recover the Rutherford formula in the low velocity limit.

Working to the lowest non-zero order in $M$ the transition amplitude becomes

$$
\mathcal{M}=-9 \pi^{3} G M \bar{u}_{s}\left(\boldsymbol{p}_{f}\right) \gamma^{0} I_{1} \gamma^{0} u_{r}\left(\boldsymbol{p}_{i}\right)
$$

where

$$
I_{1}=\int \frac{d^{3} k}{(2 \pi)^{3}} \frac{\boldsymbol{p}_{f}{ }^{2}-\boldsymbol{k}^{2}}{\left|\boldsymbol{p}_{f}-\boldsymbol{k}\right|^{7 / 2}} \frac{\not k+m}{k^{2}-m^{2}+i \epsilon} \frac{\boldsymbol{k}^{2}-\boldsymbol{p}_{i}{ }^{2}}{\left|\boldsymbol{k}-\boldsymbol{p}_{i}\right|^{7 / 2}} .
$$

Now

$$
k^{2}-m^{2}=E^{2}-\boldsymbol{k}^{2}-m^{2}=\boldsymbol{p}^{2}-\boldsymbol{k}^{2},
$$

where $E$ is the particle energy and $\boldsymbol{p}^{2}=\boldsymbol{p}_{i}{ }^{2}=\boldsymbol{p}_{f}{ }^{2}$. The pole in the propagator is therefore cancelled by the vertex factors, so there is no need for the $i \epsilon$ prescription. The integral we need to evaluate is therefore

$$
I_{1}=\int \frac{d^{3} k}{(2 \pi)^{3}} \frac{\boldsymbol{k}^{2}-\boldsymbol{p}^{2}}{\left|\boldsymbol{p}_{f}-\boldsymbol{k}\right|^{7 / 2}\left|\boldsymbol{k}-\boldsymbol{p}_{i}\right|^{7 / 2}}(\not k+m),
$$

which is evaluated in Appendix A. The result is

$$
I_{1}=\frac{1}{9 \pi^{2} \boldsymbol{q}^{2}}\left(2 m+3\left(\not p_{f}+\not \not_{i}\right)-4 E \gamma^{0}\right)
$$

where $\boldsymbol{q}=\boldsymbol{p}_{f}-\boldsymbol{p}_{i}$. It follows that

$$
\begin{aligned}
\mathcal{M} & =-\frac{\pi G M}{\boldsymbol{q}^{2}} \bar{u}_{s}\left(\boldsymbol{p}_{f}\right)\left(2 m-3\left(\not p_{f}+\not p_{i}\right)+8 E \gamma^{0}\right) u_{r}\left(\boldsymbol{p}_{i}\right) \\
& =-\frac{4 \pi G M}{\boldsymbol{q}^{2}} \bar{u}_{s}\left(\boldsymbol{p}_{f}\right)\left(2 E \gamma^{0}-m\right) u_{r}\left(\boldsymbol{p}_{i}\right),
\end{aligned}
$$


and the differential cross section is given by

$$
\frac{d \sigma}{d \Omega}=\frac{(2 G M m)^{2}}{\boldsymbol{q}^{4}}\left|\bar{u}_{s}\left(\boldsymbol{p}_{f}\right)\left(2 E \gamma^{0}-m\right) u_{r}\left(\boldsymbol{p}_{i}\right)\right|^{2} .
$$

So, despite the complexity of going to second order in the iterative solution, the result is quite straightforward. Performing the usual spin sums gives an unpolarised cross section of

$$
\begin{aligned}
\frac{d \sigma}{d \Omega} & =\frac{(G M)^{2}}{2 \boldsymbol{q}^{4}} \operatorname{Tr}\left\{\left(\not \not_{f}+m\right)\left(2 E \gamma^{0}-m\right)\left(\not p_{i}+m\right)\left(2 E \gamma^{0}-m\right)\right\} \\
& =\frac{2(G M)^{2}}{\boldsymbol{q}^{4}}\left(m^{2}\left(E^{2}-\boldsymbol{p}_{f} \cdot \boldsymbol{p}_{i}\right)+\left(2 E^{2}-m^{2}\right)^{2}+4 E^{2} \boldsymbol{p}_{f} \cdot \boldsymbol{p}_{i}\right) .
\end{aligned}
$$

If we let $v=|\boldsymbol{p}| / E$ denote the particle velocity, and $\theta$ the scattering angle, we arrive at the simple expression

$$
\frac{d \sigma}{d \Omega}=\frac{(G M)^{2}}{4 v^{4} \sin ^{4}(\theta / 2)}\left(1+2 v^{2}-3 v^{2} \sin ^{2}(\theta / 2)+v^{4}-v^{4} \sin ^{2}(\theta / 2)\right) .
$$

The formula has the satisfying property of being independent of the particle mass, as one would expect from the equivalence principle. We delay a fuller discussion of the properties of this result until after we have established that it is gauge-invariant.

\section{The Kerr-Schild Gauge}

The iterative scheme employed here suggests a generalisation to alternative field configurations. In effect, what we have done is taken the covariant Dirac equation and re-written it in the form

$$
\left.(i \not \partial-m) \psi=i \not \partial-g^{\mu} \nabla_{\mu}\right) \psi
$$

and we have interpreted the right-hand side as an interaction term $B(x)$. This method will clearly provide a sensible iterative scheme if the right-hand side contains a single factor of some power of $M$. If this is not the case, there can be no simple correspondence between the order of the iterative solution, and the order of $M$ in the amplitude. The gauge we have exploited to date has the feature that $B(x)$ goes as $M^{1 / 2}$. The obvious question now is whether we can do better and find a gauge where the interaction goes as $M$. This should then avoid having to integrate over intermediate momenta in a second order diagram. Such a gauge is provided by introducing the Eddington-Finkelstein advanced time coordinate, which can be employed to convert the Schwarzschild metric to Kerr-Schild form [14],

$$
d s^{2}=\eta_{\mu \nu} d x^{\mu} d x^{\nu}-\frac{2 G M}{r} l_{\mu} l_{\nu} d x^{\mu} d x^{\nu},
$$

where

$$
l_{\mu}=(1, x / r, y / r, z / y) .
$$


For our $g^{\mu}(x)$ matrices we choose

$$
g^{0}=\gamma^{0}+\frac{G M}{r}\left(\gamma^{0}-\gamma_{r}\right), \quad g^{i}=\gamma^{i}-\frac{G M}{r} \frac{x^{i}}{r}\left(\gamma^{0}-\gamma_{r}\right),
$$

where

$$
\gamma_{r}=\frac{x^{i}}{r} \gamma_{i}
$$

The reciprocal matrices are found to be

$$
g_{0}=\gamma^{0}-\frac{G M}{r}\left(\gamma^{0}-\gamma_{r}\right), \quad g_{i}=\gamma_{i}-\frac{G M}{r} \frac{x^{i}}{r}\left(\gamma^{0}-\gamma_{r}\right) .
$$

In this gauge the Dirac equation becomes

$$
i \not \partial \psi+\frac{i G M}{r}\left(\gamma^{0}-\gamma_{r}\right)\left(\frac{\partial}{\partial t}-\frac{\partial}{\partial r}-\frac{1}{2 r}\right) \psi=m \psi,
$$

which achieves our goal of constructing an interaction term of order $M$.

The momentum space representation of the interaction term is now

$$
B\left(\boldsymbol{p}_{2}, \boldsymbol{p}_{1}\right)=G M \int d^{3} x e^{-i \boldsymbol{p}_{2} \cdot \boldsymbol{x}} \frac{1}{r}\left(\gamma^{0}-\frac{x^{i}}{r} \gamma_{i}\right)\left(-E+i \frac{\partial}{\partial r}+\frac{i}{2 r}\right) e^{i \boldsymbol{p}_{1} \cdot \boldsymbol{x}} .
$$

The result of this integral is

$$
\begin{aligned}
B\left(\boldsymbol{p}_{2}, \boldsymbol{p}_{1}\right)= & -\frac{2 \pi G M}{|\boldsymbol{q}|^{2}}\left(4 E \gamma^{0}-\not p_{1}-\not p_{2}\right)-\frac{4 \pi G M}{|\boldsymbol{q}|^{4}}\left(\boldsymbol{p}_{2}{ }^{2}-\boldsymbol{p}_{1}{ }^{2}\right)\left(\not p_{2}-\not p_{1}\right) \\
& +\frac{i \pi^{2} G M}{|\boldsymbol{q}|^{3}}\left(\left(\boldsymbol{p}_{2}{ }^{2}-\boldsymbol{p}_{1}{ }^{2}\right) \gamma^{0}-2 E\left(\not p_{2}-\not p_{1}\right)\right) .
\end{aligned}
$$

This form of interaction is certainly not as elegant as our earlier gauge choice, but has the advantage that the first-order term in the iterative solution gives the $\mathrm{O}(M)$ contribution to the amplitude. Since the final two terms in $B\left(\boldsymbol{p}_{2}, \boldsymbol{p}_{1}\right)$ vanish on-shell, only the first term contributes to the amplitude, and we find

$$
\mathcal{M}=-\frac{4 \pi G M}{\boldsymbol{q}^{2}} \bar{u}_{s}\left(\boldsymbol{p}_{f}\right)\left(2 E \gamma^{0}-m\right) u_{r}\left(\boldsymbol{p}_{i}\right)
$$

precisely as obtained earlier.

This calculation confirms that the differential cross section formula, to order $M^{2}$, is gauge invariant. Since a series for the amplitude in this gauge can only go in orders of $M$, this suggests that there can be no $M^{3 / 2}$ contribution in the earlier gauge setup. That is, the third order term in the expansion of (21) must vanish. This is confirmed by (somewhat tedious) calculations.

\section{Discussion}

Our calculations in two distinct gauges have confirmed that, to lowest order in the black-hole mass, the unpolarised differential scattering cross section for a spin- $1 / 2$ particle is given by

$$
\frac{d \sigma}{d \Omega}=\frac{(G M)^{2}}{4 v^{4} \sin ^{4}(\theta / 2)}\left(1+2 v^{2}-3 v^{2} \sin ^{2}(\theta / 2)+v^{4}-v^{4} \sin ^{2}(\theta / 2)\right) .
$$


As already commented, this formula is independent of the particle mass and depends only on the incident velocity. This confirms that the equivalence principle is directly encoded in the Dirac equation, though it remains to be shown whether this holds to all orders. The formula also makes it clear that the low velocity limit recovers the Rutherford formula. The higher order relativistic corrections are not obvious, but do agree with the small angle formulae obtained by Collins et al. [2], who found that as $\theta \mapsto 0$ the classical cross section is given by

$$
\frac{d \sigma}{d \Omega}=\frac{4(G M)^{2}}{v^{4} \theta^{4}}\left(1+2 v^{2}+v^{4}\right) .
$$

Unlike the Collins et al. formula (their equation 12), however, there is no cubic term in $\theta^{-1}$ in the quantum result, which is an even function of $\theta$.

The massless limit $m \mapsto 0$ is also well-defined and leads to the simple formula

$$
\frac{d \sigma}{d \Omega}=\frac{(G M)^{2} \cos ^{2}(\theta / 2)}{\sin ^{4}(\theta / 2)} .
$$

Again, the low-angle limit recovers the classical formula for the bending of light. This result also predicts zero amplitude in the backward direction, $\theta=\pi$. Null geodesics produce a significant flux in the backward direction, and the fact that zero is predicted here is a diffraction effect for neutrinos which goes beyond the predictions of geometric optics. A similar prediction of zero back-scattering for neutrinos was made in [4]. A more detailed analysis of the cross section in the backward direction also reveals a large 'glory' scattering [4, 6]. In the geometric optics limit this is attributable to multiple orbits, and in the quantum description the glory scattering is described by higher-order terms in GM. To describe these effects in the present scheme requires extending to higher order in perturbation theory. This is currently under investigation.

Extending to higher orders also raises the question of the convergence of the iterative scheme proposed here. This is not a straightforward issue to address as there is no dimensionless coupling constant in the problem. Also, it is not clear whether higher-order quantum terms should still be expected to obey the equivalence principle. One can easily formulate desirable criteria for convergence, such as $G M E<1$ or $G M E v<1$, but these are too restrictive, given that the low angle formula we arrive at is expected to be valid for all masses and velocities. It would appear that the only way to investigate convergence is to compute the next order terms in the perturbation series directly.

This work should also have clarified the importance of working consistently to the correct order in $M$. This is particularly clear in the Schwarzschild gauge, where the interaction term contains factors of $1-(1-2 G M / r)^{1 / 2}$. An iterative scheme based on this gauge choice should expand out $B(x)$ as a series in $M$, and then keep all of the terms up to the desired order. Such a scheme is workable, but has the disadvantage of introducing new vertex terms at each order in the series solution. It is straightforward to confirm that such a scheme reproduces our result for the fermion cross section, to lowest order.

We can now explain the failure of the Born approximation discussed by Collins et al. [2] for the scalar case. These authors used a similar technique of viewing the difference between the true and and flat space metrics as an 
interaction term, and constructed the amplitude

$$
T\left(\boldsymbol{p}_{2}, \boldsymbol{p}_{1}\right)=2 G M \int d^{3} x e^{-i\left(\boldsymbol{p}_{2}-\boldsymbol{p}_{1}\right) \cdot \boldsymbol{x}}\left(\frac{E^{2}}{r-2 G M}+\frac{\boldsymbol{p}_{2} \cdot \boldsymbol{x} \boldsymbol{p}_{1} \cdot \boldsymbol{x}}{r^{3}}\right) .
$$

Applying this in the Born approximation as it stands produces an unphysical answer, which can be traced to the pole at the horizon. The resolution is that the correct scheme involves a series expansion of the amplitude as well so that, working to first order in $M$, we should compute

$$
T\left(\boldsymbol{p}_{2}, \boldsymbol{p}_{1}\right)=2 G M \int d^{3} x e^{-i\left(\boldsymbol{p}_{2}-\boldsymbol{p}_{1}\right) \cdot \boldsymbol{x}}\left(\frac{E^{2}}{r}+\frac{\boldsymbol{p}_{2} \cdot \boldsymbol{x} \boldsymbol{p}_{1} \cdot \boldsymbol{x}}{r^{3}}\right) .
$$

Precisely this integral is obtained in both of the the other gauges discussed in this paper, up to terms which vanish on shell. Working to first order in $M$ gives rise to the differential cross section

$$
\frac{d \sigma}{d \Omega}=\frac{(G M)^{2}}{4 v^{4} \sin ^{4}(\theta / 2)}\left(1+v^{2}\right)^{2},
$$

appropriate for a scalar field. Again, we see that the equivalence principle is obeyed, and the various low-angle and low velocity approximations are retained.

This work suggests a number of generalisations, the most obvious of which is to more general black-hole configurations. In this respect a start for the Kerr case has already been made in [9], where the Kerr solution was formulated in a gauge with similar properties to that employed in the first half of this paper. In addition, both gauges discussed here look well set up to give a proper, quantum description of radiation processes as a particle is accelerated in a gravitational field. Classical descriptions of such processes are notoriously tricky and ambiguous. A further question is whether the formalism developed here is also appropriate for the absorption problem 17, 15]. This involves modifying the vertex factor in such a way as to explicitly expose the non-Hermiticity due to the singularity. We expect to tackle these issues in future papers.

\section{A Evaluation of $I_{1}$}

To evaluate the integral $I_{1}$ of equation (32) we first displace the origin in $k$-space by $\left(\boldsymbol{p}_{f}+\boldsymbol{p}_{i}\right) / 2$ to get

$$
I_{1}=\int \frac{d^{3} k}{(2 \pi)^{3}} \frac{\left(\boldsymbol{k}+\left(\boldsymbol{p}_{f}+\boldsymbol{p}_{i}\right) / 2\right)^{2}-\boldsymbol{p}^{2}}{|\boldsymbol{q} / 2-\boldsymbol{k}|^{7 / 2}|\boldsymbol{k}+\boldsymbol{q} / 2|^{7 / 2}}\left(\not k+\left(\not p_{f}+\not p_{i}\right) / 2-E \gamma^{0}+m\right) .
$$

We now chose coordinate axis with $\boldsymbol{q}$ and $\boldsymbol{p}_{f}+\boldsymbol{p}_{i}$ defining the 3 and 1 directions respectively. These vectors are orthogonal as the momenta are on-shell. We next introduce the spheroidal coordinates

$$
\begin{aligned}
& k_{1}=\alpha \sinh u \sin v \cos \phi \\
& k_{2}=\alpha \sinh u \sin v \sin \phi \\
& k_{2}=\alpha \cosh u \cos v
\end{aligned}
$$


where $0 \leq u<\infty, 0 \leq v \leq \pi, 0 \leq \phi<2 \pi$ and $\alpha=|\boldsymbol{q}| / 2$. Exploiting the symmetry in $\boldsymbol{k}$ the integral reduces to two terms:

$$
\begin{aligned}
I_{1}= & \frac{1}{2 \pi^{2} \boldsymbol{q}^{2}} \int_{0}^{\infty} d u \int_{0}^{\pi} d v \sinh u \sin v\left\{\frac{\sinh ^{2} u-\sin ^{2} v}{\left(\sinh ^{2} u+\sin ^{2} v\right)^{5 / 2}}\left(\not p_{f}+\not p_{i}+2 m\right)\right. \\
& \left.+\frac{\sinh ^{2} u \sin ^{2} v}{\left(\sinh ^{2} u+\sin ^{2} v\right)^{5 / 2}}\left(\not \not_{f}+\not p_{i}-2 E \gamma^{0}\right)\right\}
\end{aligned}
$$

These integrals are simple to perform and lead to the result of equation (33).

\section{References}

[1] C. Itzykson and J-B. Zuber. Quantum Field Theory. McGraw-Hill, New York, 1980.

[2] P.A. Collins, R. Delbourgo, and R. M. Williams. On the elastic Schwarzschild scattering cross section. J. Phys. A, 6:161-169, 1973.

[3] N. Sanchez. Elastic scattering of waves by a black hole. Phys. Rev. D, 18(6):1798-1804, 1978.

[4] J.A.H. Futterman, F.A. Handler, and R.A. Matzner. Scattering from Black Holes. Cambridge University Press, 1988.

[5] S. Chandrasekhar. The Mathematical Theory of Black Holes. Oxford University Press, 1983.

[6] N. Andersson and B. Jensen. Scattering by black holes. gr-qc/0011025.

[7] N. Sanchez. Wave scattering and the absorption problem for a black hole. Phys. Rev. D, 16(4):937-945, 1977.

[8] A.N. Lasenby, C.J.L. Doran, and S.F. Gull. Gravity, gauge theories and geometric algebra. Phil. Trans. R. Soc. Lond. A, 356:487-582, 1998.

[9] C.J.L. Doran. New form of the Kerr solution. Phys. Rev. D, 61(6):067503, 2000 .

[10] N.D. Birrell and P. C. W. Davies. Quantum Fields in Curved Space. Cambridge University Press, 1982.

[11] M. Nakahara. Geometry, Topology and Physics. Adam Hilger, Bristol, 1990.

[12] E. Merzbacher. Quantum Mechanics. Wiley (NY), 1961.

[13] F. Mandl and G. Shaw. Quantum Field Theory. Wiley (NY), 1984.

[14] D. Kramer, H. Stephani, M. MacCallum, and E. Herlt. Exact Solutions of Einstein's Field Equations. Cambridge University Press, 1980.

[15] W.G. Unruh. Absorption cross section of small black holes. Phys. Rev. D., 14(12):3251, 1976. 